\title{
Two-Dimensional Nanomaterials for Cancer Nanotheranostics
}

\author{
Yongjiu Chen, Yakun Wu, Bingbing Sun, Sijin Liu, * and Huiyu Liu*
}

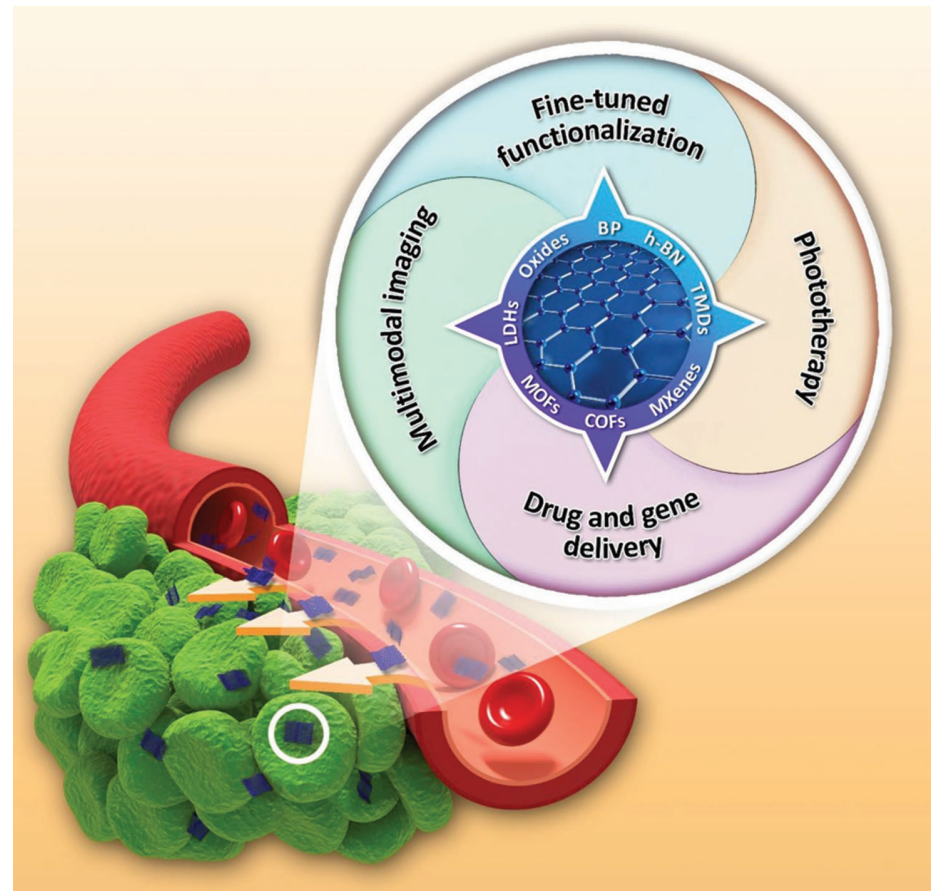

\section{From the Contents}

1. Introduction

2. Cancer Nanotheranostics using 2DNMs

3. Fine-Tuning Strategies for Better 2DNMs .....8

4. Concluding Remarks and Perspectives
$\boldsymbol{E}_{\text {merging nanotechnologies show unprecedented }}$ advantages in accelerating cancer theranostics. Among them, two-dimensional nanomaterials (2DNMs) represent a novel type of material with versatile physicochemical properties that have enabled a new horizon for applications in both cancer diagnosis and therapy. Studies have demonstrated that 2DNMs may be used in diverse aspects, including i) cancer detection due to their high propensity towards tumor markers; ii) molecular imaging for guided tumor therapies, and iii) drug and gene loading, photothermal and photodynamic cancer therapies. However, their biomedical applications raise concerns due to the limited understanding of their in vivo metabolism, transformation and possible toxicities. In this comprehensive review, the state-of-the-art development of 2DNMs and their implications for cancer nanotheranostics are presented. The modification strategies to enhance the biocompatibility of 2DNMs are also reviewed. 


\section{Introduction}

Cancer is the overarching global health burden owing to its high mortality and significant morbidity. ${ }^{[1]}$ Despite the tremendous efforts that have been devoted in the treatment of cancers, the overall five-year survival rate for cancer patients still remains poor, which necessitates the exploitation of more effective treatment and prevention strategies. ${ }^{[1]}$ With deeper investigation into the diversely dynamic and heterogeneous features of cancer, it is urgent to develop more effective "precision medicine", integrating diagnostic and therapeutic agents into one entity. Therefore, "nanotheranostics" merged as a new concept referring to the combination of diagnostic imaging and therapy. ${ }^{[2]}$ Thus, emerging elaborately designed nanomaterials offer unprecedented opportunities to treat cancer patients at a personalized level. Among them, 2DNMs quickly stand out as promising candidates in cancer diagnosis and therapy.

Since its first discovery by Geim and co-workers in 2004, ${ }^{[3]}$ graphene, the unique monolayer graphite, has sparked significant interest for the studies of $2 \mathrm{DNMs}{ }^{[3,4]}$ 2DNMs are defined as the ultrathin nanomaterials with one dimension less than $100 \mathrm{~nm} .{ }^{[5]}$ Stemming from its unique structure, 2DNMs exhibit excellent thermal, ${ }^{[6]}$ mechanical, chemical, ${ }^{[7]}$ and optical properties. ${ }^{[8]}$ These desirable properties enable considerable research activities for the applications of 2DNMs in energy storage, ${ }^{[9]}$ energy transfer, ${ }^{[10]}$ and electronic $^{[11]}$ and mechanical devices. ${ }^{[12]}$ Meanwhile, 2DNMs also harbor diverse strengths in biomedical applications, including high drug and gene loading capacity, great light heat conversion efficiency and photodynamic properties, endowing them with great potentials for use in personalized cancer nanotheranostics, such as sensing, ${ }^{[13]}$ imaging, ${ }^{[14]}$ gene and drug delivery, and therapy. ${ }^{[15]}$ For example, the advantages of rigid $\mathrm{C}-\mathrm{N}$ planes and $\mathrm{p}$-conjugated electronic structures render ultrathin $\mathrm{g}_{-} \mathrm{C}_{3} \mathrm{~N}_{4}$ nanosheets suitable for cellular nuclear twophoton fluorescence imaging. ${ }^{[16]}$ In addition, some 2DNMs have high extinction coefficients, such as $\mathrm{WS}_{2}{ }^{[17]}$ and $\mathrm{MoS}_{2}{ }^{[18]}$ offering the capabilities of effective photothermal conversion and deep tissue penetration. The large surface-to-volume ratio also enables these materials to serve as excellent carriers in drug delivery systems. ${ }^{[19]}$

Despite these novel characteristics, it still needs to go a long way toward the actual applications of 2DNMs in clinics. There are still concerns about their pharmacokinetics and toxicity. i) some 2DNMs, e.g., graphene. are easy to aggregate in physiological media, which may cause potential in vivo toxicity; ii) impurities (such as heavy metal/metal oxides obtained during the manufacture process) may be above the tolerance levels in humans; iii) slow metabolism may result in the accumulation and primary toxicities in the main organs of the human body. Thus far, many reviews have focused on the unique material properties and potential applications of 2DNMs. Some comprehensive reviews recapitulate the broad biomedical applications of nanomaterials with special focus on graphene and graphene analogues. Some reviews have covered the biological effects of graphene and its derivatives, including their ex vivo/in vivo fate and toxicity. However, to our knowledge, there is no comprehensive review that systematically profiles 2DNMs used in cancer nanotheranostics. Additionally, the strategies used to fine-tune their physicochemical properties and the biological effects of other 2D materials have not been systemically discussed thus far.Therefore, there is a notable knowledge gap concerning the physicochemical propertybases applications of 2DNMs in cancer nanotheranostics and a lack of an up-to-date summary of the applications of 2DNMs in cancer research.

In this review, we center on the topic of 2DNMs used for cancer nanotheranostics with a particular emphasis on their potential applications in cancer diagnostics, imaging and treatments, their biological effects, and fine-tuning of their physicochemical properties for the rational design of graphene materials and other 2DNMs. A summary of the representative studies published under " $2 \mathrm{D}$ " and "cancers" from 2000 to 2016, and an overview of this review are presented in Figure 1. We believe this review will inspire broader interest from related scientific fields, including the development of novel 2DNMs, the implications in cancer imaging and therapeutics, and the investigations into optimal surface modification strategies for more effective 2DNMs in cancer theranostics.

\section{Cancer Nanotheranostics using 2DNMs}

Cancer nanotheranostics incorporated in one nanoparticle has the following advantages compared with individual modality. i) The integration of imaging and therapy could provide more detailed information for precision treatment with high sensitivity and low interference. ii) A synergetic effect could be enhanced for efficient therapy. iii) The combination allows the possibility to monitor the location, size, and metastasis for different tumor stages during the whole treatment. In this section, we discuss the recent advances on 2DNMs-based nanotheranostics, which will further benefit the rational design of 2DNMs for cancer theranostics. ${ }^{[20]}$

Y. J. Chen, Y. K. Wu, Prof. S. J. Liu

State Key Laboratory of Environmental

Chemistry and Ecotoxicology

Research Center for Eco-Environmental Sciences

Chinese Academy of Sciences

Beijing 100085, China

E-mail: sjliu@rcees.ac.cn

Prof. B. B. Sun

School of Chemical Engineering

Dalian University of Technology

Dalian 116024, China

Prof. H. Y. Liu

Beijing Key Laboratory of Bioprocess

Beijing Advanced Innovation Center for Soft Matter Science and Engineering

Beijing Laboratory of Biomedical Materials

Beijing University of Chemical Technology

Beijing 100029, China

E-mail: liuhy@mail.buct.edu.cn

DOI: $10.1002 / \mathrm{smll} .201603446$ 


\subsection{Synthesis and Fundamental Properties of 2DNMs}

Since the historical wake of graphene in 2004, 2DNMs have become the most exploited nanomaterials due to their unique physicochemical properties. Their ultrathin two-dimensional structure endows them with many exceptional features, ${ }^{[21]}$ including electromechanical modulation, ${ }^{[22]}$ extremely high carrier mobility, ${ }^{[23]}$ tunable band gap, ${ }^{[24]}$ and quantum Hall effect. ${ }^{[25]}$ The reliable and reproducible synthesis of 2DNMs is the prerequisite for their physicochemical characterization and their integration with multiple bioapplications. To date, many methods have been reported to manipulate 2DNMs with various shapes, dimensions and quality. They are mainly divided into two strategies, namely top-down and bottom-up strategies. The former approach is to directly exfoliate layered crystals via external driving forces, e.g., mechanical or chemical exfoliation. Typical methods of the latter approach include chemical vapor deposition (CVD) growth and wet chemistry. Here we briefly discuss 4 main fabrication methods (mechanical exfoliation, epitaxial growth, CVD and wet chemistry) of 2DNMs, and more details can be found elsewhere. ${ }^{[26]}$

Mechanical exfoliation is the original method to generate graphene nanosheets. Many atomically thin 2DNMs can be fabricated using this method, e.g., carbon nitride, [27] hexagonal boron nitride $(\mathrm{h}-\mathrm{BN}),{ }^{[28]} \mathrm{MoS}_{2},{ }^{[8 \mathrm{~b}]} \mathrm{WSe}_{2},{ }^{[29]}$ and $\mathrm{WS}_{2} \cdot{ }^{[8 \mathrm{~b}, 30]}$ Using adhesive tape, single or a few layers of pristine 2DNMs can be produced and deposited on $\mathrm{Si}$ or $\mathrm{SiO}_{2}$ substrates for further electronic studies. Geim's laboratory successfully prepared various $2 \mathrm{DNMs}$, including $\mathrm{MoS}_{2},{ }^{[30]}$ boron nitride $(\mathrm{BN}),{ }^{[31]}$ and $\mathrm{NbSe}_{2},{ }^{[32]}$ leading to the winning of the Nobel Prize in Physics (2010). Although this "scotchtape" approach is an easy way to obtain high-quality 2DNMs for small scale laboratory research purposes, it is not suitable for large-scale industrial production. To solve this problem, an epitaxial growth method is employed to produce graphene by a high temperature graphitization on insulator or metal substrates such as $\mathrm{SiC}, \mathrm{SiO}_{2}, \mathrm{Ni}(111)$ and $\operatorname{Ir}(111) .{ }^{[33]}$ Although the graphitization of $\mathrm{SiC}$ dates back to several decades ago, ${ }^{[34]}$ only recent work has demonstrated that it is possible to produce a large quantity of graphene for electronic applications.

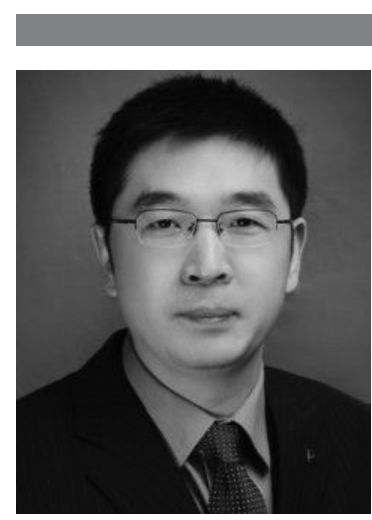

Sijin Liu received his $\mathrm{Ph} . \mathrm{D}$. degree from the Institute of Developmental Biology and Genetics, CAS, in 2004. After postdoctoral training in hematology and oncology at MIT and Children's Hospital Boston, Harvard Medical School from 2004 to 2008, he became an instructor at Tufts Medical School from 2008. Dr. Liu moved to the Research Center for Eco-Environmental Sciences, the Chinese Academy of Sciences (CAS) as a professor at the end of 2009. Dr. Liu is the chief scientist of "National Basic Research Program of China (973 Program)". His research interests focus on, a) nanoimpact; b) environmental cancer biology.

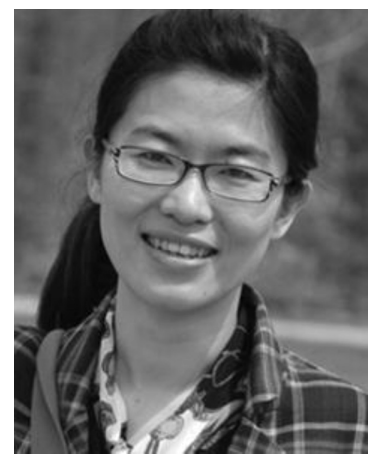

Huiyu Liu received her Ph.D. Degree from the Technical Institute of Physics and Chemistry (TIPC), the Chinese Academy of Sciences (CAS), in 2007. Thereafter, she worked as an associate professor at TIPC. Dr. Liu moved to Beijing University of Chemical Technology as a full professor at the end of 2015. Her research mainly focuses on nanomedicine and nanobiology, including the safe design of smart nanoparticle-based platforms for cancer therapy, the characterization and understanding of the biological effects of nanomaterials with important implications in human health.

Besides the epitaxial growth, CVD is an alternative and robust method for large-scale fabrication of high quality graphene. The CVD reaction is a catalytic reaction between catalytic metal substrates and hydrocarbon gases. This technology dates back to 1973 for a monolayer growth of semiconductors, insulators, or magnetic materials, and the strategy is commercially available now. ${ }^{[35]}$ However, this method still suffers from some weaknesses, such as limitations of the choices for appropriate substrates on which to grow the desired material, the requirements for high temperatures,
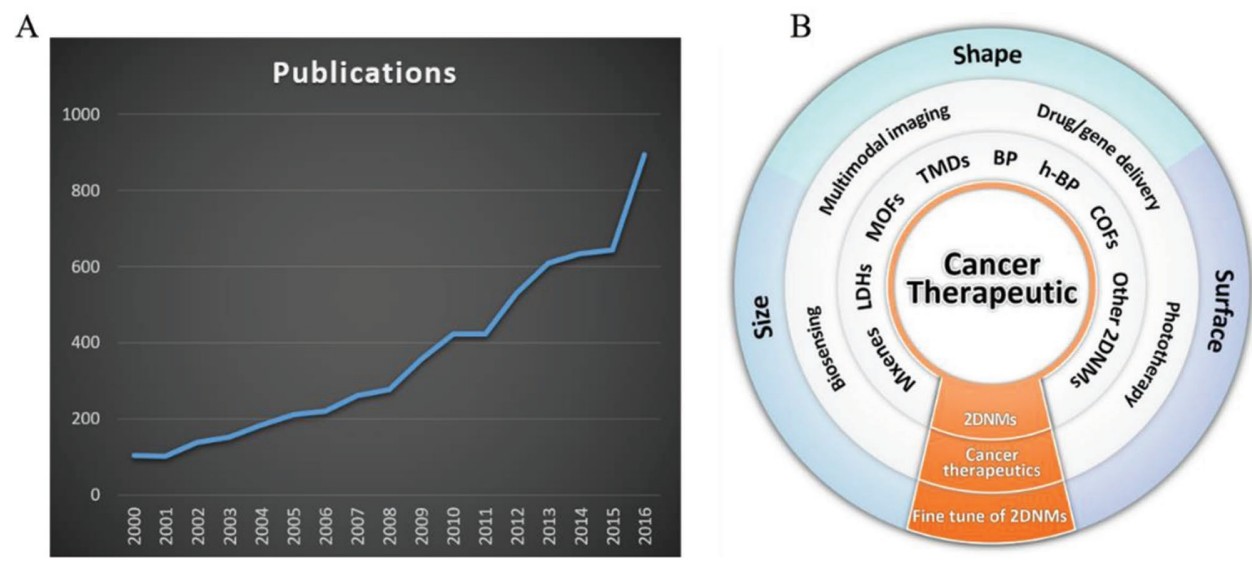

Figure 1. A) The number of publications under the research topics of " $2 D$ " and "cancers" from 2000 to 2016 identified in Pubmed. B) A diagram depicting the structure of the current review. This review focuses on cancer diagnostics and therapeutics. 2DNMs have been used in cancer fine diagnostics and therapeutics (e.g., biosensing, multimodal imaging, phototherapy, drug/gene delivery) and 2DNMs can be fine-tuned by size, shape, surface and other strategies such as targeting ligands. 


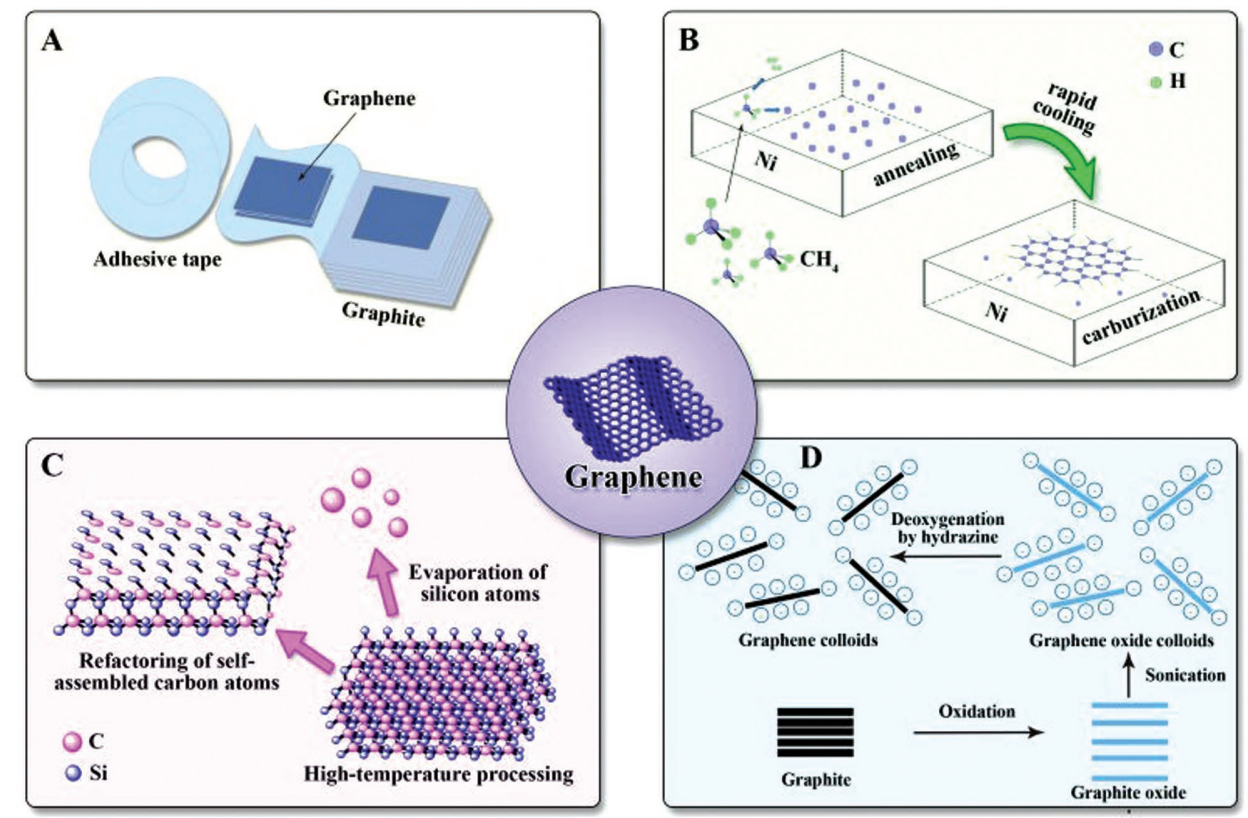

Figure 2. Typical synthesis methods of graphene. A) Mechanical exfoliation of graphite, B) CVD method, C) epitaxial growth on SiC, and D) wet chemistry.

critical vacuum conditions, and removal of products from the substrates at the final step. Furthermore, the CVD method is suitable for producing single-crystalline 2D nanodevices for electronic applications, but not for biomedical applications.

Another mainstream method to fabricate graphene and graphene oxide $(\mathrm{GO})$ is the solution-processing method. Using an extreme oxidation process, all of these methods give rise to the same aqueous dispersion of GO flakes with hydrophilic functional groups. ${ }^{[36]}$ Compared with the CVD method, the solution-processing method is more suitable for preparing 2DNMs for biomedical purposes due to the following reasons, i) to have the desirable functional groups with acceptable choices of solvents and surfactants during the preparation; ii) to obtain relatively small flakes for long circulation in physiological conditions. These established methods have commendably augmented the development of 2DNMs. The aforementioned 4 different methods for the fabrication of graphene as the shining surrogate of 2DNMs are summarized in Figure 2.

Besides strategies discussed above, doping also has also recently drawn the attention of many researchers. Diverse metal and nonmetal elements are doped into 2DNMs, such as $\mathrm{N}^{\left[{ }^{[37}\right]} \mathrm{ZnO},{ }^{[38]}$ or metal ions $\left(\mathrm{Mg}^{2+}, \mathrm{Zn}^{2+}, \mathrm{Ca}^{2+}, \mathrm{Al}^{3+}\right) \cdot{ }^{[39]}$ These doped 2DNMs not only enhance the electrochemical activity in bioimaging and biosensing, ${ }^{[37,40]}$ but also strengthen the interaction between 2DNMs and loaded drugs. Moreover, many novel nontoxic organics are integrated into 2DNMs, such as polymethylmethacrylate, ${ }^{[41]}$ polyvinylpyrrolidone (PVP),${ }^{[42]}$ and carboxylic acid. ${ }^{[43]}$ These strategies will increase the biocompatibility of 2DNMs to achieve longer half-times in circulation.

\subsection{Biosensing of Tumor Biomarkers}

Tumor biomarkers are defined as the native substances or harvested by tumors or in tumor microenvironments in light of tumorigenesis and progression, including hormones, enzymes, proteins, and oncogene. They are considered as a convincing tool to monitor cancer progression, such as diagnosis and treatment. Early detection of specific tumor markers, such as carcino-embryonic antigen (CEA), alpha fetal protein (AFP) and p53, is a determinant factor for treatability, curability and long-term prognosis. For 2DNMs, their unique merits confer them with remarkable capabilities as the probes to monitor the tumor biomarkers. For example, the large surface of 2DNMs guarantees the immobilization of various biosensing molecules for an easy hybridization between target substances and labeled probes (Figure 3). Besides, fluorescent quenching may occur due to photo-induced electron transfer between 2DNMs and fluorophore, which is also useful in some optical biosensing. ${ }^{[44]}$

Many 2DNMs-based biosensors rely on their interactions with single-strand DNA (ssDNA). 2DNMs can be considered as an energy acceptor or quencher that suppresses fluorophore-labeled ssDNA via energy transfer. When ssDNA is hybridized with its complementary chain and detached from 2DNMs, the fluorescence gets recovered. For instance, Yuan and colleagues synthesized layered $\mathrm{WS}_{2}$ by a one-step procedure for a quenching-and-recovery sensing. [45] In this study, a $\mathrm{WS}_{2}$ nanosheet acted as the dark quencher, and ssDNA labeled with a fluorescent tetramethylrhodamine (TAMRA) was used as the probe. This $\mathrm{WS}_{2}$-based DNA sensor manifested remarkable sensitivity with the limit of detection (LOD) of $60 \mathrm{pM}$, better than GO-based and $\mathrm{MoS}_{2}$-based biosensors. ${ }^{[45]}$ This finding suggests that $\mathrm{WS}_{2}$ can act as a "nanolab" to adsorb such biomolecules as peptides and oligonucleotides, and can be applied as a reversible "mix-and-detect" assay probe. Similar to CEA, AFP also plays an important role in tumor development. In 2013, Zhao and co-workers designed an enzyme-free electrochemical sensor for the detection of AFP based on sandwich-like 

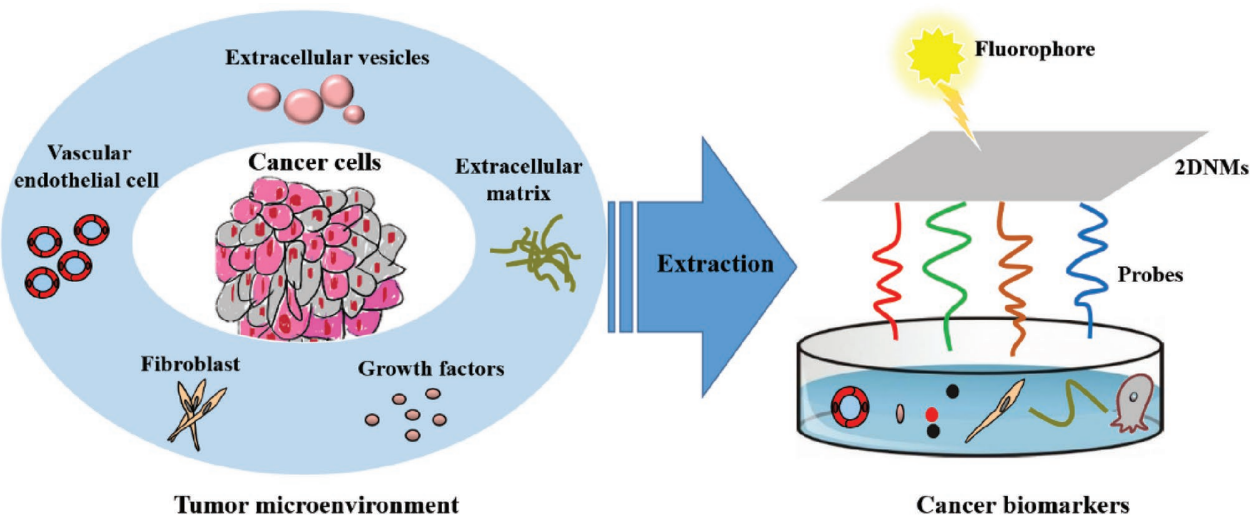

Figure 3. A scheme of cancer biomarkers derived from tumor microenvironment or excreted into blood, such as extracellular vesicles, extracellular matrix, growth factors. 2DNMs are applied for the monitoring of cancer biomarkers, by the immobilization with various probes (DNA/RNA/protein) as well as the fluorophore.

structures that were composed of Au-Pd nanocrystals and $\mathrm{N}$-doped graphene. Their results exhibited a favorable LOD at $0.005 \mathrm{ng} \mathrm{mL}^{-1}$, as well as linear range of $0.05-30 \mathrm{ng} \mathrm{mL}^{-1}$ as well. ${ }^{[46]}$ The high-efficient catalysis of Au-Pd nanocrystals towards $\mathrm{H}_{2} \mathrm{O}_{2}$ and enhanced electron transfer of graphene would guarantee an extremely low LOD and ultrahigh sensitivity.

P53 is a crucial tumor suppressor that governs cell growth and death and also regulates DNA repair. ${ }^{[47]}$ Different from the AFP detection mechanism, p53 can be monitored via a multi-enzymatic amplification strategy. ${ }^{[48]} \mathrm{Du}$ et al. reported a new multienzyme sensor through the conjugation of $\mathrm{p} 53^{392}$ signal antibody $\left(\mathrm{p} 53^{392} \mathrm{Ab}_{2}\right)$ and horseradish peroxidase (HRP) on GO (namely HRP-p $53^{392} \mathrm{Ab}_{2}$-GO). In the presence of hydrogen peroxide $\left(\mathrm{H}_{2} \mathrm{O}_{2}\right)$ and thionine, ${ }^{[48]}$ HRP-p $53^{392} \mathrm{Ab}_{2}$-GO captured onto the electrode can amplify the electrocatalytic process through reduction of oxidized thionine. With the large surface of $\mathrm{GO}$ for $\mathrm{Ab}_{2}$ loading via amine condensation or electrochemical interaction, the cascade amplification between $\mathrm{Ab}_{2}$ and phospho-p53 played an important role in this system. ${ }^{[48]}$ Thus, the results provided a clear demonstration of an ultra-sensitive and accurate quantitative determination of phosphorylated $\mathrm{p} 53$, with the LOD at $0.01 \mathrm{nM}$ and a board linear range of $0.02-2 \mathrm{nM}$. ${ }^{[48]}$

\subsection{Multimodal Imaging}

The predominant imaging technologies in cancer therapeutics include computer tomography (CT), photoacoustic tomography (PAT), positron emission tomography (PET), magnetic resonance imaging (MRI), and optical imaging (OI) ${ }^{[49]}$ All these technologies have been developed and greatly improved in close conjunction with nanoscience and nanotechnology (Figure 4). ${ }^{[20 b, 50]}$ Before appreciating the role of 2DNMs in bioimaging, we should not ignore their special characteristics. The optical properties are one of their most attractive features. For example, graphene is has the property of non-fluorescence due to the absence of bandgap. However, some graphene-based nanomaterials can produce strong photoluminescence owing to the generation of energy bandgaps when they were nanosized or have

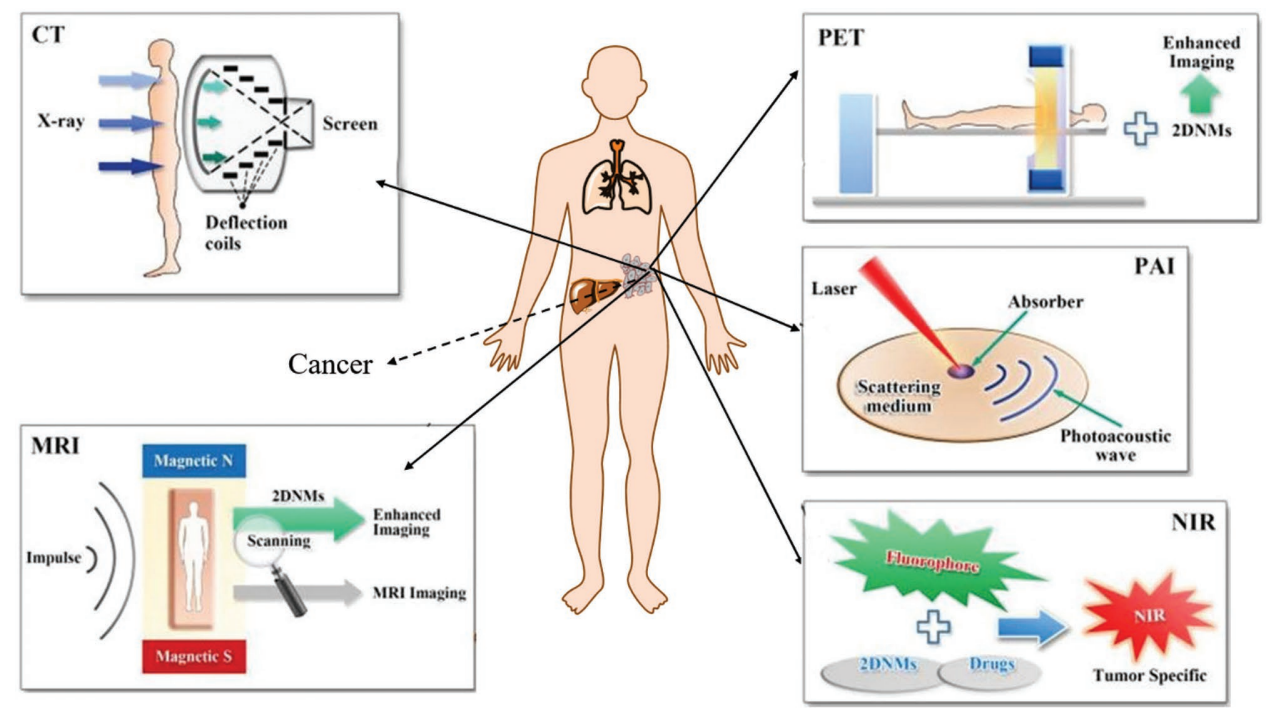

Figure 4. Schematic of imaging techniques used to visualize tumors in humans. Imaging technologies are growing with the aid of nanotechnology, including CT, MRI, PAT/PAI, NIR imaging, and PET. 
some defects, which is the consequence of the quantum confinement effect. ${ }^{[51]}$ Another property is the above-discussed fluorescence-quenching, deriving from nonradiative dipoledipole interactions or fluorescence resonance energy transfer (FRET) between 2DNMs and fluorescent probes. ${ }^{[51 b]}$ This property is not only favorable for 2DNMs-based DNA sensors, but also benefits the resonance Raman spectroscopy to suppress the interference of fluorescence. ${ }^{[52]}$ Besides, 2DNMs harbor many merits, such as easy surface engineering, high electron mobility, high surface area and biocompatibility. In line with these properties, 2DNMs are becoming popular candidates for cancer-targeted imaging. ${ }^{\text {[20b] }}$

CT is the most common method used in cancer imaging, where there is scanning and conversion through a digital matrix by a computer after irradiation with X-rays. Wang et al. fabricated $\mathrm{MoS}_{2} / \mathrm{Bi}_{2} \mathrm{~S}_{3}$-PEG nanosheets for multimodal imaging and therapy. The $\mathrm{MoS}_{2}$ framework processes good PAT imaging and high photothermal conversion efficiency, while Bi harbors high X-ray attenuation in CT imaging as well as photoelectric absorbance ability for the generation of secondary electron enhancing radiation therapy. Their study also revealed that the $\mathrm{MoS}_{2} / \mathrm{Bi}_{2} \mathrm{~S}_{3}$-PEG nanosheets showed reasonable biocompatibility without notable toxicity for 40 days after intravenous injection on the basis data from hematology, histology, and body weight. ${ }^{[53]}$

Due to the hazards posed by the radioactivity of X-rays, FI imaging using small dye molecules is an attractive alternative for cancer treatment. A study carried out by Kim and colleagues constructed a platform for the conjugation of IR825 (a NIR dye), PEG and hyaluronic acid to reduced graphene oxide (rGO) surface. Near-infrared fluorescence was thereafter applied to distinguish the retention, efficacy, and permeability of drugs at cancer sites. ${ }^{[54]}$ Some dualimaging methods, such as NIR-FI, can guide in vivo photothermal therapy (PTT) when the used 2DNMs show marked photoelectron absorption and photothermal conversion. For example, Yang and colleagues designed a complex of PEGylated nano-graphene sheets with fluorescent molecule cyanine7 (Cy7), for a successful in vivo FI of tumors in mice and ex vivo FI of organs after intravenous administration. ${ }^{[55]}$

Recent advances also demonstrate the benefits of 2DNMs in PAT. When a photoabsorbent material is exposed to a short-pulse laser, the material can convert the luminous energy into heat. ${ }^{[56]}$ After this conversion, the temperature is elevated in local tissues, leading to the expansion of local exposure and production of pressure waves that can be detected by ultrasonic detectors. ${ }^{[57]}$ PAT has some characteristics different from MRI and FI, such as strong penetration, a high resolution ratio and non-invasive feature. ${ }^{[58]}$ Upon administration of $\mathrm{MoS}_{2}$-IO-(d)PEG $\left(\mathrm{MoS}_{2}=0.68 \mathrm{mg} / \mathrm{mL}\right)$ into 4T1 tumor-bearing mice, PAT imaging was performed at different time points post administration. PAT imaging provided strong signals, with a time dependent retention of 2DNMs after in vivo administration into tumor sites. ${ }^{[59]}$ A similar study showed that PEG-coated $\mathrm{WS}_{2}$ can be used in both CT and PAT imaging. ${ }^{[60]}$ While CT and MRI offer clear contrast for tumor imaging, the strong photoacoustic signals have higher spatial resolution and sensitivity. More importantly, the photoacoustic signals provide estimable parameters that contribute to detailing the tumor microenvironment and the distribution of nanomaterials. ${ }^{[60]}$ In addition to the great resolution of PAT, the imaging depth of PAT is also outstanding. ${ }^{[61]}$ One study showed that $\mathrm{rGO}$ generated a clear photoacoustic signal 3 times greater than that of $\mathrm{GO}$ under identical conditions. ${ }^{[62]}$ After transplanting MCF-7 tumor xenografts into mice, PAT signals yielded clearer images than the control group with the same irradiation source at $808 \mathrm{~nm}, 2 \mathrm{~h}$ after administration of nano-rGO. ${ }^{[62]}$ The wide scope of PAT applications includes not only tissue and tumor in situ imaging, but also the visualization of organelles and cells. ${ }^{[61]}$ This high resolution of PAT makes it favorable for functional and metabolic analyses of vessels, micro-anatomy, and small molecules. Besides, functional photoacoustic microscopy can acquire high resolution with the help of the photoacoustic effect. ${ }^{[63]}$ Zhang and co-workers took advantage of the photoacoustic effect and developed a hybrid strategy for tumor imaging, especially for the visualization of hemoglobin oxygen saturation, which contributed to the vivid imaging of tumor blood vessels. ${ }^{[64]}$

In contrast, PET, another emerging imaging technology, has not been extensively studied in cancer nanotheranostics. Of note, PET can detect the decayed positrons of radioactive isotopes, and most nanomaterials are labeled with their corresponding isotope, such as ${ }^{64} \mathrm{Cu} \cdot{ }^{[65]}$ Frank et al. reviewed isotope labeling, while Raman spectroscopy acts as an effective method for the synthesis of carbon isotope labeled nanographene. ${ }^{[6]}$ Before the application of carbon isotope labeled graphene in PET imaging, ${ }^{64} \mathrm{Cu}$ labeled magnetism nanomaterials, such as iron oxide, were demonstrated to indicate high imaging capabilities in tumor diagnosis. When ${ }^{64} \mathrm{Cu}$-iron oxide nanoparticles were administrated in tumor-harboring mice, PET imaging revealed an apparent contrast. ${ }^{[67]}$ Thus, it could be assumed that 2D-nanomaterials might be possibly used in PET imaging for cancer therapeutics.

\subsection{Drug and Gene Delivery}

Nanomaterials are expected to outperform chemotherapy and radiation therapy in cancer therapy due to their finetuned drug delivery and release. Of them, 2DNMs exhibit a powerful capability in drug and gene delivery with many strengths (Figure 5). One of the unique essential features of 2DNMs is the lamella structure that ensures the vast surface area for high-efficiency of drug/gene loading. ${ }^{[4]}$ Moreover, 2DNMs and their derivatives can strongly interact with anti-cancer drugs through supramolecular $\pi-\pi$ stacking and hydrophobic interactions owing to their numerous surface contacts and special $\mathrm{sp}^{2}$-bond of carbon atoms. ${ }^{[68]}$

$\mathrm{GO}$ and rGO have been verified to be efficient carriers of genetic macromolecules, contrast agents, ${ }^{[68]}$ and drugs. Different methods for drug/gene loading and delivery have been assessed in vivo and ex vivo. Covalent bonding of many functional molecules, such as chitosan, ${ }^{[69]}$ folic acid (FA), ${ }^{[70]}$ or PEG onto GO sheets will generate a platform for drug loading to deliver hydrophilic anti-cancer agents, such as doxorubicin (DOX). ${ }^{[71]}$ PEG functionalized GO shows more stable properties than pristine $\mathrm{GO}$ in different solution 
A



B

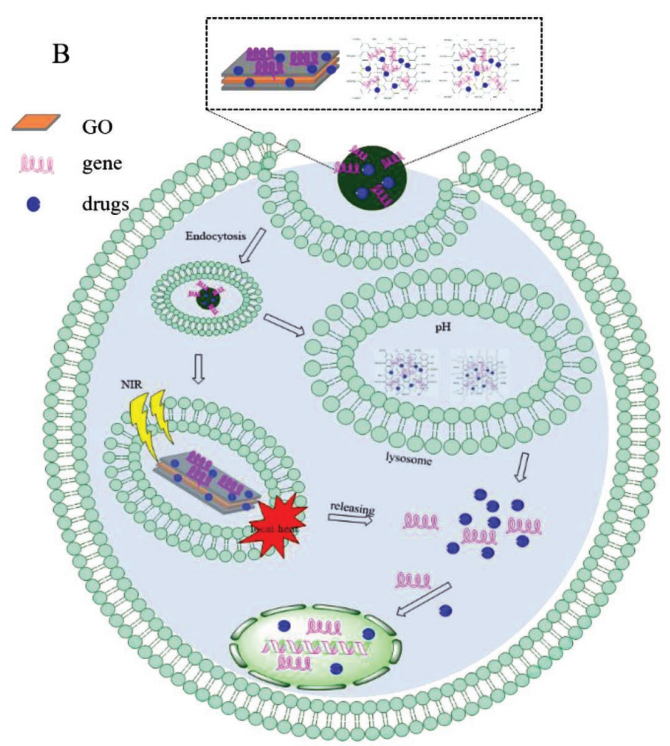

Figure 5. Schematic of drug and gene delivery using 2DNMs as carriers. A) Loading genes or drugs onto (1) 2DNMs (e.g., GO); (2) PEG-modified 2DNMs; (3) hybrid 2DNMs. B) Delivery of genes/drugs into cells.

conditions, such as phosphate buffered solution (PBS), serum, and cell culture media as well. PEG-modified GO can form complexes in the presence of aromatic molecules, an antineoplastic drug SN-38. ${ }^{[72]}$ A PEG-GO/SN-38 complex showed no significant difference in drug release compared to free SN-38 dispersed in dimethyl sulfoxide (DMSO). However, it has a 1000 times better curative efficiency than that of the conventional anti-tumor drug CPT in HCT-116 cells. ${ }^{[72]} \mathrm{A}$ similar study carried by Yang et al. showed that DOX could be loaded onto GO approximately $2.35 \mathrm{mg} \mathrm{mg}^{-1}$ while the initial concentration of DOX is $0.47 \mathrm{mg} \mathrm{mL}^{-1} .^{[73]}$ Moreover, the loading and release efficiency of DOX on GO is strongly dependent on $\mathrm{pH}$, owing to the hydrogen bonding between GO surface and the attached DOX.

Graphitic-phase carbon nitride $\left(\mathrm{g}-\mathrm{C}_{3} \mathrm{~N}_{4}\right)$, a novel 2D semiconductor material, reveals spectacular characteristics in photo-catalysis and electro-catalysis. ${ }^{[7]}$ At the same time, its favorable homogeneity, dramatic hydrophilicity, and imperceptible toxicity, are highly desirable biomedical applications. Recently, highly dispersed $\mathrm{g}-\mathrm{C}_{3} \mathrm{~N}_{4}$ nanosheets were synthesized via ultrasonic exfoliation by chemical oxidation of bulk g- $\mathrm{C}_{3} \mathrm{~N}_{4} \cdot{ }^{[75]}$ The as-prepared $\mathrm{g}-\mathrm{C}_{3} \mathrm{~N}_{4}$ is able to deliver anti-cancer drugs (DOX), as well as acting as a photodynamic therapeutic approach. It is impressive that the DOX loading efficacy can reach more than $18000 \mathrm{mg} \mathrm{g}^{-1}$. The efficiency of DOX released from $\mathrm{g}-\mathrm{C}_{3} \mathrm{~N}_{4}$ nanosheets depended on the local $\mathrm{pH}$ value, where increased acidity accelerated the release of the DOX from the carriers. ${ }^{[75]}$

In addition to $\mathrm{GO}$ and its analogues, transition metal oxide nanomaterials of 2DNMs also act as a loading and releasing system for drugs or genes in cancer therapeutics. Chen et al. built a $\mathrm{pH}$ mediated drug-releasing $\mathrm{MnO}_{2}$ nano-lamella, where DOX would rapidly released from $\mathrm{MnO}_{2}$ in response to a low $\mathrm{pH}$ value. ${ }^{[76]}$ With the modified surface charge from $-40 \mathrm{mV}$ to $+22 \mathrm{mV}$, DOX can be successfully conjugated onto $\mathrm{MnO}_{2}$ nanosheets. ${ }^{[76]}$ Furthermore, ex vivo cellular assays indicated that DOX-loaded $\mathrm{MnO}_{2}$ nanostructures could enhance the anti-cancer outcomes and also reduce the multi-drug resistance of cancer cells. ${ }^{[76]}$

Besides the individual drug loading and delivery, tumor targeted ligands and anti-cancer drugs have also been rapidly developed. Wang and colleagues successfully developed a system called GSPID that is a mesoporous silica-coated GO nanosheet, loaded with DOX and targeting peptide-IP (IL-13 peptide). ${ }^{[7]}$ The IP modification remarkably enhanced the aggregation of DOX into glioma cells, ${ }^{[77]}$ and thus improved the effectiveness in cancer therapeutics.

In addition to the delivery of drugs to tumor locations, approaches for gene delivery are also developed for cancer therapeutics. The aim of gene delivery is to transport nucleic acid molecules to cytoplasm or nucleus, ${ }^{[78]}$ and to exert their therapeutic functions. ${ }^{[79]}$ On one hand, nucleic acid molecules (such as small RNA molecules) that are integrated onto 2DNMs can directly inhibit the growth of cancer cells through the mechanism of gene expression silencing. ${ }^{[80]}$ On the other hand, 2DNMs were also studied for gene transfection $^{[81]}$ and reporter gene assay. ${ }^{[82]}$ Furthermore, gene delivery was investigated in conjunction with other theranostics, including PAT, ${ }^{[83]}$ PTT, ${ }^{[83]}$ and other methods. ${ }^{[84]}$

\subsection{Phototherapy}

Phototherapy includes PTT and photodynamic therapy (PDT). Photothermal therapy, which employs a laser to generate heat and to induce hyperthermia in local tumor sites, would cause protein denaturation and cell membrane disruption and thus incur inevitable cancer cell death. ${ }^{[85]}$ However, due to the poor selectivity and high power density of the ray, it will bring destory to normal and cancer cells, and lead to adverse effects. ${ }^{[86]}$ The importation of a photothermal agent (PTA) will improve the targeting accuracy by confining the laser power to local tumor sites with the use of a relatively low laser power density. ${ }^{[6]}$ To this end, 2DNMs have been 
widely studied in PTA applications for both ex vivo and in vivo photothermal depletion of cancer cells.

To enhance the therapeutic selectivity and to achieve accurate tumor targeting, several groups have developed tumor-targeting GO nanosheets for PTT. Dai's group used noncovalently PEGylated nano-rGO with the targeting peptide Arg-Gly Asp (RGD) for selective photothermal killing of U87MG cancer cells. ${ }^{[87]}$ In another study, Akhavan et al. showed that an ultralow concentration of PEGylated GO nanoribbons functionalized with RGD peptide could be used for targeted PTT. ${ }^{[88]}$ In addition, the same group also recently developed an efficient in vivo photothermal agent using RGD-functionalized rGO nanomeshes in a mouse model. ${ }^{[89]}$ These GO nanomeshes were thereafter functionalized with PEG, RGD and Cy7, and were thus used for in vivo cancer targeting and FI of cancers in mice. The rGO nanomesh-PEG solution exhibited approximately 5- and 22-fold higher NIR absorption under $808 \mathrm{~nm}$ irradiation than rGO-PEG and pristine GO, respectively. ${ }^{[88,90]}$

Inspired by these encouraging studies, researchers further explored graphene analogues, such as layered molybdenum disulfide $\left(\mathrm{MoS}_{2}\right)$ in cancer phototherapy. ${ }^{[8 \mathrm{~b}]}$ Irradiation at diverse wavelengths to monitor ${ }^{[91]}$ and to trigger ${ }^{[92]}$ biological events were investigated in bioimaging and PTT modalities. For example, photothermal ablation makes use of NIRabsorbing materials to transduce light into thermal energy. ${ }^{[92]}$ The subsequent thermal energy can be applied to a number of purposes, including cancer ablation and drug release. In spite of the intensive interest in PTAs, the greater depth development of PTT has been facing considerable challenges, such as the limited absorption profiles required for NIR photothermal transduction.

Chou and colleagues performed chemical exfoliation on $\mathrm{MoS}_{2}\left(\mathrm{ceMoS}_{2}\right)$ for NIR PTAs, which can be easily constructed in abundant batches. Moreover, $\operatorname{ceMoS}_{2}$ can be directly synthesized in hydrophilic conditions. ${ }^{[6]}$ This work showed that $\mathrm{CeNS}_{2}$ heated up rapidly upon NIR irradiation. Due to its high surface to volume ratio, $\mathrm{ceMoS}_{2}$ possessed a strong loading capacity on GO. ${ }^{[6]}$ Furthermore, Liu and colleagues prepared $\mathrm{MoS}_{2}$ nanosheets with the chemically exfoliated method and with modification of lipoic acid and terminated polyethylene glycol (LA-PEG) to generate PEGylated $\mathrm{MoS}_{2}\left(\mathrm{MoS}_{2}\right.$-PEG), which is more stable in different solutions than naked $\mathrm{MoS}_{2} \cdot{ }^{[93]}$ Utilizing the vast surface area of PEG modified $\mathrm{MoS}_{2}$, the photodynamic agent, Ce6, can be loaded on the obtained $\mathrm{MoS}_{2}-\mathrm{PEG}$ through physical adsorption. Increased cellular uptake of $\mathrm{MoS}_{2}-\mathrm{PEG} /$ Ce6 remarkably improved the photodynamic therapeutic efficacy. ${ }^{[93]}$ Wang et al. thereafter proposed a high-efficiency and succinct method for the controlled construction of PEGmodified $\mathrm{MoS}_{2}$ nanosheets on the basis of the "bottom-up" strategy containing both atoms. The distinct PEG-mediated solvothermal procedure generates $\mathrm{MoS}_{2}$ nanosheets with better control of size, crystallinity, and colloidal stability. Furthermore, to obtain the optimized photothermal performance of $\mathrm{MoS}_{2}$ nanosheets, the material is functionalized through modulating the lateral and lamella size as well as surface modification. PEGylated $\mathrm{MoS}_{2}$ nanosheets with the superior performance of photothermal conversion are utilized for highly efficient PTT of tumors in animal models. Moreover, the optimized $\mathrm{MoS}_{2}$-PEG nanosheets exhibit an encouraging anti-cancer efficacy without observable ex vivo and in vivo hemolysis, coagulation and toxicity. ${ }^{[94]}$ Likewise, Liu's study demonstrated the likelihood of $\mathrm{WS}_{2}$ as a highly effective strategy for in vivo tumor ablation by photothermal therapeutic in mice. ${ }^{[95]} \mathrm{WS}_{2}$ nanosheets also possess a preferential multifunctional structure for both bioimaging of cancer and PDT-PTT for cancer eradication, which would be a synergistic cancer therapy. ${ }^{[96]}$ Their work demonstrated that combined phototherapies of PDT-PTT showed about threefold of the efficacy in cancer cell ablation compared to individually PTT or PDT strategy, with the methylene blue serving as a photosensitizer. ${ }^{[96]}$

Furthermore, it was found that $2 \mathrm{D} \mathrm{Bi}_{2} \mathrm{Se}_{3}$ also processed a capability to convert a NIR laser into thermal effect for PTT. ${ }^{[97]}$ In Li's study, PVP-coated 2D $\mathrm{Bi}_{2} \mathrm{Se}_{3}$ nanoplates were synthesized with $90 \mathrm{~nm}$ of lateral size and $4 \mathrm{~nm}$ of thickness. This ultra-small structure of $\mathrm{PVP}-\mathrm{Bi}_{2} \mathrm{Se}_{3}$ revealed a rapid temperature increase to $50.2{ }^{\circ} \mathrm{C}$ within 5 min following $808 \mathrm{~nm}$ laser irradiation with low power density. Tumors were completely eradicated after intratumor injection of the material. ${ }^{[97]}$ Additionally, polymer/BN nanocomposite materials have also been reported to have superior thermal transport performance, and may be a potential candidate for the use as a PTA in cancer therapy, ${ }^{[98]}$ which requires further detailed investigation.

In addition to the popularity of PTT in cancer therapeutics, PDT also plays an important role. PDT is a photosensitizer that is stimulated under a specific wavelength to transform energy into the nearby oxygen, which results in the generation of reactive oxygen species (ROS). ${ }^{[99]}$ Furthermore, ROS will react with biomolecules to produce cytotoxicity to kill cancer cells. ${ }^{[99 a]}$ After the first PDT application in the malignant tumor therapy by Diamond and co-workers ${ }^{[100]}$ and the precursory work of GO as the photosensitizer, ${ }^{[99}$ a] more and more researchers started to utilize the novel optoelectronic properties of 2DNMs for cancer ablation. ${ }^{[101]}$ Compared to the conventional PDT, 2DNMs can be used as a platform not only for the intrinsic PDT, but also as the loading vehicle of photosensitizer [99b,102] and targeting ligands. ${ }^{[103]}$ Wang and co-workers constructed a layered double hydroxide (LDH) for the supporting of both $\mathrm{Ce} 6$ and $\mathrm{Pt}($ IV), leading to a significant increase in cell death for A2780cisR cells. ${ }^{[104]}$ Moreover, the combination of PTT and PDT is very promising in cancer treatment. ${ }^{[93]}$ Cao's group developed a GO-PEG-Ce6 system for both PTT and PDT to restrain 4T1 breast cancer cells, and their results revealed extensive damage to tumor tissues. ${ }^{[105]}$ Thus, the diverse configuration of 2DNMs with superior optical-electrical characteristics and vast surfaces can be used for efficient cancer therapeutics through different mechanisms. ${ }^{[96,106]}$

\section{Fine-Tuning Strategies for Better 2DNMs}

While there have been rapid developments in 2DNMs's applications, our understanding of their toxicity and biological 
impact struggled to keep pace. In general, unmodified 2DNMs, such as graphene, GO, $\mathrm{rGO}, \mathrm{MoS}_{2}$, and $\mathrm{LDH}$, exhibit severe toxicity to the body. For example, naked 2DNMs may disturb the heart rate, hatching efficacy, angiogenesis, and morphogenesis in a zebrafish model. ${ }^{[107]}$ Pristine 2DNMs can provoke serious systemic inflammation, ${ }^{[108]}$ acute injury, ${ }^{[109]}$ or even death in mic. ${ }^{[110]}$ These toxicities are closely related to the physicochemical properties of 2DNMs, such as size, ${ }^{[111]}$ surface charge, ${ }^{[112]}$ modifications, ${ }^{[113]}$ and exposure factors including dosage, ${ }^{[114]}$ time, ${ }^{[114,115]}$ and route. ${ }^{[108,114,116]}$ Thus, fine-tuning the intrinsic properties of 2DNMs and modulating exposure factors are critical to improve the biocompatibility and theranostics efficacy.

\subsection{Optimizing of Shape, Size and Surface (3S) Strategies}

When 2DNMs are exposed to biosystems, it's generally agreed that $3 \mathrm{~S}$, including their shape, size and surface properties, could directly influence their in vivo toxicity by affecting their circulation, distribution, and excretion. Here we will focus on these factors for a deep understanding of the rules influencing the properties of 2DNMs, and the strategies for improvement.

\subsubsection{Effects of Size and Shape of 2DNMs on Biocompatibility}

In a general sense, the size of 2DNMs refers to both the lateral and vertical sizes that influence their biocompatibility and the functionality in biological systems. Our recent work has evaluated the effect of lateral sizes (S-GO: >95\% 50-350 nm; I-GO: mostly $350-750 \mathrm{~nm}$; L-GO: nearly $750-1300 \mathrm{~nm}$ ) on the generation of pro-inflammatory responses in cells. We demonstrated that S-GO were mainly phagocytized by macrophages, while L-GO nanosheets were adsorbed on the membrane and activated the pro-inflammatory responses. Furthermore, L-GO stimulated in vivo M1 polarization of macrophages, associated with the ample generation of inflammatory cytokines and pronounced recruitment of pro-inflammatory cells. ${ }^{[111 b]}$ These findings suggest that fine-tuning lateral size would be a sound strategy to improve the biocompatibility of 2DNMs. ${ }^{[111 b]}$

Due to the maturation and toleration of normal somatic cells to 2DNMs, stem cells are employed for more sensitive toxicity evaluation for 2DNMs. Akhavan and co-workers ${ }^{[117]}$ prepared reduced GO nanoplatelets (rGONPs) after the sonication of $\mathrm{rGO}$ sheets. These results showed that the $11 \mathrm{~nm}$ rGONPs could enter the nuclei of stem cells and induce genotoxicity after $1 \mathrm{~h}$ treatment, whereas $\mathrm{rGO}$ showed no remarkable toxicity after $24 \mathrm{~h}$ exposure. ${ }^{[117]}$

\subsubsection{Surface Modification for Better Performance of 2DNMs}

To improve the biocompatibility and increase the time of circulation of 2DNMs in blood, a variety of modification approaches were adopted for functionalization of nanomaterials. A common modification for enhancing the biocompatibility of 2DNMs is PEGylation. PEG is a type of water-soluble polymer and non-toxic to cells, rendering the 2DNMs to combine with many organic substances. ${ }^{[118]}$ Mounting evidence has shown that PEG-modified 2DNMs are more stable with greater dispersion not only in water and PBS but also in cell culture medium. ${ }^{[72-93]}$ For example, in a study by Yang and co-workers, no significant toxicity was observed in animals 90 days after administration of PEGylated graphene, and histological examination of organ sections also revealed no significant injuries. ${ }^{[119]}$ A similar study by Liu and colleagues demonstrated that GO coated with PEG did not obviously spin down after centrifugation at $10000 \mathrm{~g}$ for 5 minutes in several solutions (water, PBS, cell medium, and serum). ${ }^{[72]}$ In contrast to the direct single modification of nanomaterials, combinatory modifications are also proved to be feasible and beneficial. ${ }^{[98]}$

Other strategies besides PEGylation have also been explored for enhanced stability and improved efficacy in cancer theranostics. For example, GO is loaded with small molecules, such as dotarem, prohance, and magnevist. ${ }^{[120]}$ Hung and co-workers illustrated that modification using small molecules could strengthen the drug delivery and GO adsorption via hypothetical $\pi-\pi$ stacking and hydrophobic effects. To obtain better theranostics, a wide usage of ligands, including proteins, PEG, FA, antibodies, glucose, DNA, RNA, cells, and bacteria, have been developed to gain better biological activity and/or effective in vivo targeting.

Technologies have been applied for mitochondrial and nucleic targeting. Since mitochondria are curial for cell survival, including in ATP generation, heme synthesis, and regulation of cell death, ${ }^{[121]}$ dual drug targeting of mitochondria and nuclei is a potent approach for cancer therapy. Mallick et al. developed this approach for dual drug delivery using $\alpha$-tocopheryl succinate to damage mitochondria, and cisplatin and DOX to destroy DNA in nuclei subcellular of HeLa cells. ${ }^{[122]}$ By targeting organelles, direct lethal damage to cancer cells at the genetic and metabolic levels would become more efficient. ${ }^{[123]}$

\subsection{Intracorporal Process of 2 DNMs}

Since the exposure factors influence the metabolism of nanomaterials, the absorption, bio-distribution, metabolism, and excretion (ADME) of 2DNMs will be an important consideration in the field of toxicology. Different properties of 2DNMs dictate different uptake, distribution, and excretion mechanisms.

After the uptake of 2DNMs, different exposure routes may determine different bio-distribution. Oral administration of ${ }^{125} \mathrm{I}-\mathrm{PEG}-\mathrm{GO}$ and ${ }^{14} \mathrm{C}$-graphene resulted in the accumulation of materials in stomach, but limited GO in intestine. ${ }^{109,124]}$ This shows that these materials cannot be abundantly adsorbed by the digestive system. Inhalation administration led to localization of ${ }^{125} \mathrm{I}-\mathrm{GO}$ in the lung, which easily caused chronic pulmonary toxicity and acute lung injury. ${ }^{[16 a]}$ Mao and co-workers showed that after intratracheal instillation of graphene for 4 weeks, $47 \%$ of the graphene retained in lung, less than $1 \%$ remained in spleen and liver. ${ }^{[109]}$ These data provide an evidence that graphene 
deposition in tissue will incur injuries. Intraperitoneal injection is another common way to expose 2DNMs in a mouse model. Kurantowicz's group demonstrated that plenty of 2DNMs, especially graphene, intruded on the peritoneum and mesenterium 4 weeks post-intraperitoneal injection. ${ }^{[125]}$ Additionally, intravenous injection is a commonly used administration route of $2 \mathrm{DNMs}$ in mice. $2 \mathrm{DNMs}$ are first localized to liver, spleen and lung by the reticulo-endothelial system (RES) and mononuclear phagocyte system (MPS). ${ }^{[13,119]}$ In contrast, smaller GO (less than $40 \mathrm{~nm}$ ) would cross the glomerular and get rapidly excreted, ${ }^{[126]}$ while middle-sized GO (100-500 nm) tended to accumulate in the liver and large GO (several micrometers) appeared to localize in the lungs upon the intravenous administration. ${ }^{[127]}$ Furthermore, since the hepatic portal vein plays a vital role in blood circulation, nanomaterials seemingly localize into the liver, and then redistribute to different organs through blood circulation, including cancer tissue. ${ }^{[127]}$ These findings suggest that different size and modified ligands of 2DNMs will promote specific tissue toxicities. All together, these data illustrate that the distribution of nanomaterials is closely determined by the exposure routes, thus a proper administration strategy would improve therapeutic efficacy for 2DNMs.

As for metabolism and excretion of 2DNMs, limited studies has investigated the in vivo chemical transformation process. A study carried out by Pieper and co-workers showed that GO could generate ROS; however, it did not influence the total amount of intracellular ROS. This work also revealed that the toxicity of pristine GO stemmed from the endoperoxides. This finding may explain why pristine GO poses significant toxicity, which could be attributed to free radicals generated by the intrinsic surface endoperoxide groups. ${ }^{[128]}$ Another important aspect should be considered in intracorporal process of 2DNMs is the excretion. Many studies have demonstrated that most 2DNMs entering the body were excreted by urine or feces over a long-time period. ${ }^{[14,119,125]}$ A study by Yang and co-workers showed that PEGylated graphene was excreted from urine more than feces, and chemical (1,4,7,10-tetraazacyclododecane-1,4,7,10tetraacetic acid) functionalized ${ }^{111}$ In-GO showed more excretion from urine than feces. ${ }^{[126]}$ In contrast, Kanakia et al. demonstrated that modified $\mathrm{GO}$ nanosheets with dextran was excreted by feces more than urine after $48 \mathrm{~h}$ intravenous administration. ${ }^{[114]}$ These results suggest that differentially modified or functionalized ligands determine excretion ways. Further studies should be carried out to find out whether a specific chemical modification group favors the excretion by urine or feces, and to access the possible toxicity of 2DNMs in the digestive and urinary systems.

Thus far, most studies have focused on the ADME of carbon 2DNMs, especially the bio-distribution of graphene, GO and rGO, while limited studies offer solid data on other 2DNMs, such as $\mathrm{MoS}_{2}$ and BP. Thus, we should pay more attention to the novel 2DNMs with better biocompatibility for applications in cancer theranostics.

\section{Concluding Remarks and Perspectives}

Cancer nanotheranostics have grown at a fast rate in recent years. Since the pioneering work of Yang in 2010, ${ }^{[129]}$ 2DNMs have been showing great potential for applications in cancerrelated fields during the whole treatment process spanning from diagnosis to therapy. Their noteworthy characteristics include high surface area, specific planar morphology, and other physicochemical properties (Figure 6). Despite superior properties and diverse biological activities of 2DNMs, most 2DNMs have emerged for only a few years and remain still in their infancy, and there are considerable challenges for the actual use of 2DNMs in clinical practices.

i) For synthesis requirements, depending on the purpose of applications, each application has differential demands. 2DNMs for biological applications should harbor desired

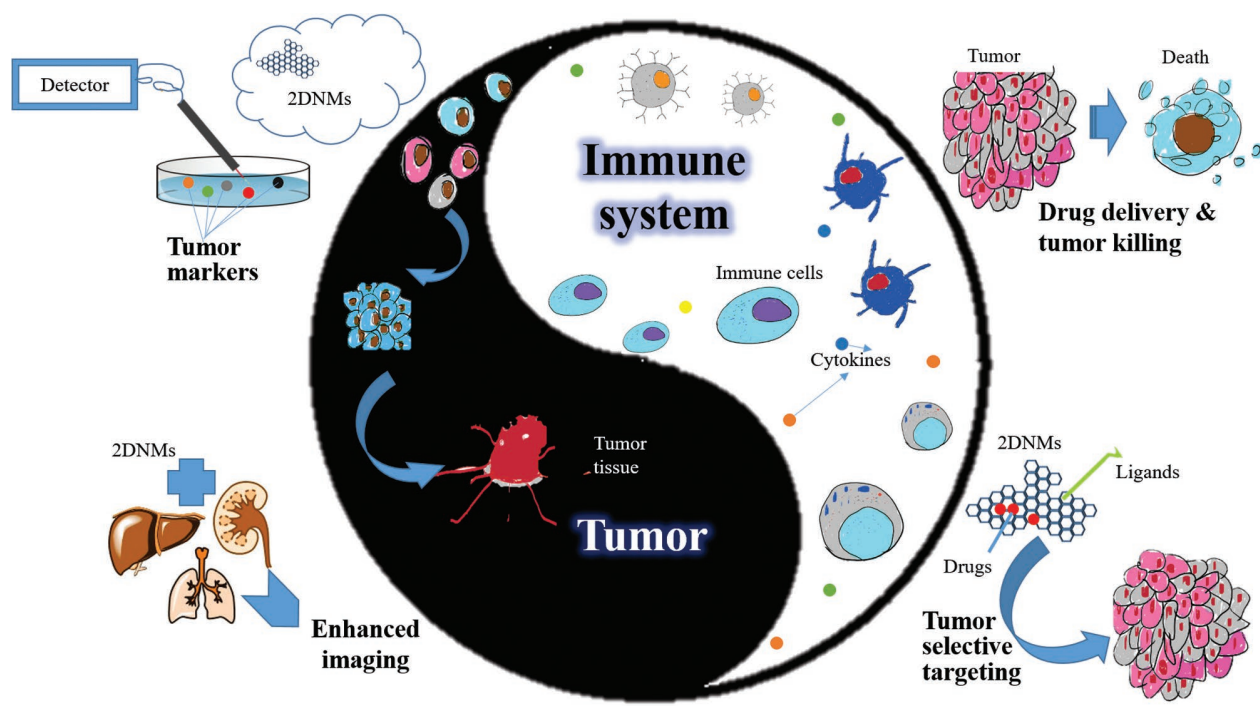

Figure 6. A scheme delineating the applications of 2DNMs cancer nanotheranostics. On one hand, 2DNMs can be used for detection of cancer cells, bio-imaging, drug delivery vehicles and therapeutics agents. On the other hand, 2DNMs can also be used as immunopotentiators to enhance the anti-cancer effects through immune cell recruitment and secretion of cytokines. 
compositions and structures, such as fine-tuned size and shape, hydrophilicity, and reasonable dissolution characteristics. For short-term nanoformulas, there is a trend to generate nanovehicles with a typical size range between 20 and $500 \mathrm{~nm}$. The particles should be small enough to go through the gaps of the tumor vasculature but large enough to avert the escape from the tumor. However, the lateral sizes of most 2DNMs are generally in the range from sub-micrometer to a few micrometers. Thus, especially for those 2DNMs exfoliated by the top-down method, it is not easy to obtain products with a narrow and even lateral size distribution. The bottom-up method may complement, but may be impeded by the scale-up problem. Therefore, it is a major obstacle to optimize the desired structures of 2DNMs with applicable production.

ii) Another concern is that multiple functions integrated in a single nanoparticle may weaken the performance of each modality. Till now, previous studies have been less concerned with a thorough understanding of the complexities, including the loading ratio of drug and imaging agents, non-specific binding, aggregations, and the loss of biological activities upon transition from ex vivo to in vivo. Thus, it is rather important to justify the ratio of drug loading, and to equilibrate the functionalities against complexities. Standard protocols should be established for smart particle design with harmonization between properties and functions, which will eventually improve their long-term performance.

iii) For cancer theranostics, "precision medicine" will continue to be a cutting-edge topic. Most 2DNMs mainly accumulate in the liver and spleen after opsonization and phagocytosis by MPS. Therefore, how to precisely target tumor sites with evasion of RES trapping is a big challenge for 2DNMs' clinical translation. Enhanced permeability and retention (EPR) effect and target binding are two main strategies. To enhance time-dependent EPR, an appropriate size is a prerequisite, as discussed above. Besides, hydrophobicity is used to increase the tumor's retention; however, 2DNMs with no hydrophilic surfaces will be rapidly taken up by RES. To obtain prolonged circulation, 2DNMs' surface must be modified through a "cloaking" strategy, like PEGylation ${ }^{[94]}$ or zwitterionic-coatings. ${ }^{[130]}$ It should be noted that hypoxic and necrotic regions exist in many tumor types, which are not easily reached by 2 DNMs. We need to bear this in mind when designing passive targeting. For active targeting, the conjugation of antibodies or antibody fragments and enzymes will efficiently guide the delivery to the targeted sites. Nevertheless, this process requires multiple copies of targeting ligands, which may promote immunogenicity and result in opsonization-mediated clearance. ${ }^{[131]}$ To this end, more efforts should be devoted to understanding the cellular and molecular mechanisms responsible for target binding and also the customized design of 2DNMs.

iv) Another important issue is the toxicology and nanoimpact of 2DNMs. From our point of view, three aspects should be considered. First, degradation should be considered. Degradability will endow nanomedicine with an ability to excrete through renal filtration, by which to reduce liver toxicity. Some 2DNMs, like GO and $\mathrm{MoS}_{2}$, are recognized via oxidative degradation in biological settings. For example, GO revealed a peroxidase enzyme-dependent oxidative degradation. ${ }^{[132]} \mathrm{MoO}_{\mathrm{x}}$ was reported to be oxidized into $\left[\mathrm{Mo}^{\mathrm{VI}} \mathrm{O}_{4}\right]^{2-}$ ions or ultra-small nanoparticles for further renal clearance. ${ }^{[133]}$ Other 2DNMs are almost chemically inert, implying that they are difficult to metabolize or degrade. Thus, developing biodegradable 2DNMs is an open direction. Second, ADME is a consideration. For clinical applications, short-term cellular cytotoxicities are not desired, although they are highly informative. Collective data of long-term tracking and tailored ADME information of these customized 2DNMs are highly desired. Third, secondary toxicity is also a big challenge. The concern that has been overlooked in the past is the secondary toxicity and low-dosage adverse effects. Thus far, the majority of research has looked into the primary toxicology of 2DNMs, and much less attention has been put on the secondary toxicity and low-dose adverse effects, especially in the long term. Additionally, the fate and potential impact of the materials that are phagocytized by cancer cells and phagocytes remain largely unknown. Thus, hunting for preferential 2DNMs with seemly biocompatibility and apt modification strategies will expedite the employment of 2DNMs in cancer therapeutics.

In the end, we highly recommend that other resources, such as genomic data, system maps and high throughput screening technologies, could be incorporated into this field. Despite many unresolved problems, we believe that 2DNMs with rational functionalities and sound biosafety profiles will surely move towards future translations.

\section{Acknowledgments}

Y.J.C. and Y.K.W. contributed equally to this work. This work was supported by grants under the national "973" program (grant number: 2014CB932000, 2016YFA0201500), the Strategic Priority Research Program of the Chinese Academy of Sciences (Grant No. XDB14000000), grants from the National Natural Science Foundation of China (grant numbers: 21425731, 21377159, 21637004, 51572271 and 31271075), the Fundamental Research Funds for the Central Universities (buctrc201610, JD1609).

[1] F. Bray, A. Jemal, N. Grey, J. Ferlay, D. Forman, Lancet Oncol. 2012, 13, 790 .

[2] D. Sun, Mol. Pharm. 2010, 7, 1879.

[3] K. S. Novoselov, A. K. Geim, S. V. Morozov, D. Jiang, Y. Zhang, S. V. Dubonos, I. V. Grigorieva, A. A. Firsov, Science 2004, 306, 666.

[4] a) H. Zhang, ACS Nano 2015, 9, 9451; b) X. Huang, Z. Y. Zeng, H. Zhang, Chem. Soc. Rev. 2013, 42, 1934.

[5] C. Chung, Y.-K. Kim, D. Shin, S.-R. Ryoo, B. H. Hong, D.-H. Min, Acc. Chem. Res. 2013, 46, 2211. 
[6] S. S. Chou, B. Kaehr, J. Kim, B. M. Foley, M. De, P. E. Hopkins, J. Huang, C. J. Brinker, V. P. Dravid, Angew. Chem. Int. Ed. 2013 , $52,4160$.

[7] a) Y. Q. Guo, K. Xu, C. Z. Wu, J. Y. Zhao, Y. Xie, Chem. Soc. Rev. 2015, 44, 637; b) C. L. Tan, H. Zhang, J. Am. Chem. Soc. 2015, $137,12162$.

[8] a) J. X. Liang, S. B. Tang, Z. X. Cao, J. Phys. Chem. C 2011, 115, 18802; b) H. Matte, A. Gomathi, A. K. Manna, D. J. Late, R. Datta, S. K. Pati, C. N. R. Rao, Angew. Chem. Int. Ed. 2010, 49 , 4059.

[9] a) Y. F. Sun, S. Gao, Y. Xie, Chem. Soc. Rev. 2014, 43, 530; b) X. Huang, C. L. Tan, Z. Y. Yin, H. Zhang, Adv. Mater. 2014, 26, 2185; c) Q. P. Lu, Y. F. Yu, Q. L. Ma, B. Chen, H. Zhang, Adv. Mater. 2016, 28, 1917; d) X. Zhang, Z. C. Lai, C. L. Tan, H. Zhang, Angew. Chem. Int. Ed. 2016, 55, 8816; e) G. Z. Sun, X. Zhang, R. Z. Lin, J. Yang, H. Zhang, P. Chen, Angew. Chem. Int. Ed. 2015, 54, 4651; f) G. Z. Sun, J. Q. Liu, X. Zhang, X. W. Wang, H. Li, Y. Yu, W. Huang, H. Zhang, P. Chen, Angew. Chem. Int. Ed. 2014, 53, 12576

[10] J. Li, C. H. Lu, Q. H. Yao, X. L. Zhang, J. J. Liu, H. H. Yang, G. N. Chen, Biosens. Bioelectron. 2011, 26, 3894.

[11] a) L. L. Peng, X. Peng, B. R. Liu, C. Z. Wu, Y. Xie, G. H. Yu, Nano Lett. 2013, 13, 2151; b) C. L. Tan, Z. D. Liu, W. Huang, H. Zhang, Chem. Soc. Rev. 2015, 44, 2615; c) H. Li, J. Wu, Z. Y. Yin, H. Zhang, Acc. Chem. Res. 2014, 47, 1067.

[12] J. J. Miao, M. Miyauchi, T. J. Simmons, J. S. Dordick, R. J. Linhardt, J. Nanosci. Nanotechnol. 2010, 10, 5507.

[13] a) J. Chao, M. Zou, C. Zhang, H. F. Sun, D. Pan, H. Pei, S. Su, L. H. Yuwen, C. H. Fan, L. H. Wang, Nanotechnology 2015, 26, 1; b) C. L. Tan, P. Yu, Y. L. Hu, J. Z. Chen, Y. Huang, Y. Q. Cai, Z. M. Luo, B. Li, Q. P. Lu, L. H. Wang, Z. Liu, H. Zhang, J. Am. Chem. Soc. 2015, 137, 10430; c) Y. Zhang, B. Zheng, C. F. Zhu, X. Zhang, C. L. Tan, H. Li, B. Chen, J. Yang, J. Z. Chen, Y. Huang, L. H. Wang, H. Zhang, Adv. Mater. 2015, 27, 935.

[14] O. Rabin, J. Manuel Perez, J. Grimm, G. Wojtkiewicz, R. Weissleder, Nat. Mater. 2006, 5, 118

[15] B. J. Hong, O. C. Compton, Z. An, I. Eryazici, S. T. Nguyen, ACS Nano 2012, 6, 63 .

[16] X. D. Zhang, H. X. Wang, H. Wang, Q. Zhang, J. F. Xie, Y. P. Tian, J. Wang, Y. Xie, Adv. Mater. 2014, 26, 4438.

[17] X. Z. Cui, Z. G. Zhou, Y. Yang, J. Wei, J. Wang, M. W. Wang, H. Yang, Y. J. Zhang, S. P. Yang, Chin. Chem. Lett. 2015, 26, 749.

[18] W. Feng, L. Chen, M. Qin, X. J. Zhou, Q. Q. Zhang, Y. K. Miao, K. X. Qiu, Y. Z. Zhang, C. L. He, Sci. Rep-UK. 2015, 5,

[19] a) K. Yang, L. Z. Feng, Z. Liu, Adv. Drug. Delivery Rev. 2016, 105, 228; b) Y. Yang, A. M. Asiri, Z. Tang, D. Du, Y. Lin, Mater. Today 2013, 16, 365.

[20] a) Y. Zhao, N. N. Li, F. Z. Yuan, H. D. Zhang, S. J. Xia, J. Appl. Polym. Sci. 2016, 133, 43988; b) M. Orecchioni, R. Cabizza, A. Bianco, L. G. Delogu, Theranostics 2015, 5, 710; c) V. P. Chauhan, R. K. Jain, Nat. Mater. 2013, 12, 958.

[21] M. C. Hersam, ACS Nano 2015, 9, 4661.

[22] J. S. Bunch, A. M. van der Zande, S. S. Verbridge, I. W. Frank, D. M. Tanenbaum, J. M. Parpia, H. G. Craighead, P. L. McEuen, Science 2007, 315, 490.

[23] M. Notarianni, J. Z. Liu, K. Vernon, N. Motta, Beilstein J. Nanotechnol. 2016, 7, 149.

[24] J. R. Feng, G. C. Wang, Comput. Mater. Sci. 2016, 111, 366.

[25] K. S. Novoselov, Z. Jiang, Y. Zhang, S. V. Morozov, H. L. Stormer, U. Zeitler, J. C. Maan, G. S. Boebinger, P. Kim, A. K. Geim, Science 2007, 315, 1379

[26] a) M. M. Fan, Z. Q. Feng, C. L. Zhu, X. Chen, C. T. Chen, J. Z. Yang, D. P. Sun, J. Mater. Sci. 2016, 51, 10323; b) M. Daud, M. S. Kamal, F. Shehzad, M. A. Al-Harthi, Carbon 2016, 104, 241.

[27] J. S. Zhang, Y. Chen, X. C. Wang, Energy Environ. Sci. 2015, 8, 3092.
[28] J. O. Island, G. A. Steele, H. S. J. c. d. Zant, A. Castellanos-Gomez, Sci. Lett. J. 2015, 4, 1.

[29] H. Li, G. Lu, Y. L. Wang, Z. Y. Yin, C. X. Cong, Q. Y. He, L. Wang, F. Ding, T. Yu, H. Zhang,Small 2013, 9, 1974.

[30] T. Georgiou, R. Jalil, B. D. Belle, L. Britnell, R. V. Gorbachev, S. V. Morozov, Y. J. Kim, A. Gholinia, S. J. Haigh, O. Makarovsky, L. Eaves, L. A. Ponomarenko, A. K. Geim, K. S. Novoselov, A. Mishchenko, Nat. Nanotechnol. 2013, 8, 100.

[31] R. V. Gorbachev, I. Riaz, R. R. Nair, R. Jalil, L. Britnell, B. D. Belle, E. W. Hill, K. S. Novoselov, K. Watanabe, T. Taniguchi, A. K. Geim, P. Blake, Small 2011, 7, 465.

[32] Y. Cao, A. Mishchenko, G. L. Yu, E. Khestanova, A. P. Rooney, E. Prestat, A. V. Kretinin, P. Blake, M. B. Shalom, C. Woods, J. Chapman, G. Balakrishnan, I. V. Grigorieva, K. S. Novoselov, B. A. Piot, M. Potemski, K. Watanabe, T. Taniguchi, S. J. Haigh, A. K. Geim, R. V. Gorbachev, Nano Lett. 2015, 15, 4914.

[33] a) K. V. Emtsev, A. Bostwick, K. Horn, J. Jobst, G. L. Kellogg, L. Ley, J. L. McChesney, T. Ohta, S. A. Reshanov, J. Rohrl, E. Rotenberg, A. K. Schmid, D. Waldmann, H. B. Weber, T. Seyller, Nat. Mater. 2009, 8, 203; b) X. J. Xing, Y. Zhou, X. G. Liu, D. W. Pang, H. W. Tang, Small 2014, 10, 3412; c) J. C. Meyer, A. K. Geim, M. I. Katsnelson, K. S. Novoselov, T. J. Booth, S. Roth, Nature 2007, 446, 60.

[34] D. V. Badami, Nature 1962, 193, 569.

[35] J. J. Tietjen, Annu. Rev. Mater. Sci. 1973, 3, 317.

[36] V. C. Tung, M. J. Allen, Y. Yang, R. B. Kaner, Nat. Nanotechnol. 2009, 4, 25.

[37] W. M. Si, W. Lei, Q. L. Hao, X. F. Xia, H. Zhang, J. Li, Q. H. Li, R. M. Cong, Electrochim. Acta 2016, 212, 784.

[38] J. Liu, Z. Y. Zhang, Y. Y. Lv, J. F. Yan, J. N. Yun, W. Zhao, L. Kou, C. X. Zhai, Compos. Part. B-Eng 2016, 99, 366.

[39] F. L. Theiss, G. A. Ayoko, R. L. Frost, Appl. Surf. Sci. 2016, 383, 200

[40] S. G. Wang, Z. T. Cui, M. H. Cao, Electrochim. Acta 2016, 210, 328.

[41] R. F. Wei, X. L. Tian, H. Zhang, Z. L. Hu, X. He, Z. Chen, Q. Q. Chen, J. R. Qiu, J. Alloys Compd. 2016, 684, 224.

[42] X. Zhi, H. L. Fang, C. C. Bao, G. X. Shen, J. L. Zhang, K. Wang, S. W. Guo, T. Wan, D. X. Cui, Biomaterials 2013, 34, 5254.

[43] W. Zhang, RSC Adv. 2015, 5, 103649.

[44] Y. Chen, C. L. Tan, H. Zhang, L. Z. Wang, Chem. Soc. Rev. 2015, 44, 2681.

[45] Y. X. Yuan, R. Q. Li, Z. H. Liu, Anal. Chem. 2014, 86, 3610

[46] L. F. Zhao, S. J. Li, J. He, G. H. Tian, Q. Wei, H. Li, Biosens. Bioelectron. 2013, 49, 222

[47] F. Toledo, G. M. Wahl, Nat. Rev. Cancer 2006, 6, 909.

[48] D. Du, L. M. Wang, Y. Y. Shao, J. Wang, M. H. Engelhard, Y. H. Lin, Anal. Chem. 2011, 83, 746.

[49] X. Li, X. N. Zhang, D. X. Li, J. Chang, Cancer. Biol. Med. 2016, 13, 339.

[50] J. Lin, X. Y. Chen, P. Huang, Adv. Drug. Deliver. Rev. 2016, 105, 242.

[51] a) C. Xin, J. X. Zheng, Y. T. Su, S. K. Li, B. K. Zhang, Y. C. Feng, F. Pan,J. Phys. Chem. 2016, 120, 22663; b) H. F. Dong, W. C. Gao, F. Yan, H. X. Ji, H. X. Ju,Anal. Chem. 2010, 82, 5511.

[52] L. M. Xie, X. Ling, Y. Fang, J. Zhang, Z. F. Liu, J. Am. Chem. Soc. 2009, 131, 9890

[53] S. G. Wang, X. Li, Y. Chen, X. J. Cai, H. L. Yao, W. Gao, Y. Y. Zheng, X. An, J. L. Shi, H. R. Chen, Adv. Mater. 2015, 27, 2775.

[54] S. H. Kim, J. E. Lee, S. M. Sharker, J. H. Jeong, I. In, S. Y. Park, Biomacromolecules 2015, 16, 3519.

[55] K. Yang, S. Zhang, G. X. Zhang, X. M. Sun, S. T. Lee, Z. Liu, Nano Lett. 2010, 10, 3318.

[56] G. Lalwani, X. Cai, L. Nie, L. V. Wang, B. Sitharaman, Photoacoustics 2013, 1, 62

[57] C. Kim, C. Favazza, L. H. V. Wang, Chem. Rev. 2010, 110, 2756.

[58] J. P. Zhong, S. H. Yang, X. H. Zheng, T. Zhou, D. Xing, Nanomedicine 2013, 8, 903. 
[59] T. Liu, S. X. Shi, C. Liang, S. Shen, L. Cheng, C. Wang, X. J. Song, S. Goel, T. E. Barnhart, W. B. Cai, Z. Liu, ACS Nano 2015, 9, 950.

[60] L. Cheng, J. J. Liu, X. Gu, H. Gong, X. Z. Shi, T. Liu, C. Wang, X. Y. Wang, G. Liu, H. Y. Xing, W. B. Bu, B. Q. Sun, Z. Liu, Adv. Mater. 2014, 26, 1886.

[61] L. H. V. Wang, S. Hu, Science 2012, 335, 1458.

[62] Z. H. Sheng, L. Song, J. X. Zheng, D. H. Hu, M. He, M. B. Zheng, G. H. Gao, P. Gong, P. F. Zhang, Y. F. Ma, L. T. Cai, Biomaterials 2013, 34, 5236.

[63] T. Sun, G. J. Diebold, Nature 1992, 355, 806.

[64] H. F. Zhang, K. Maslov, G. Stoica, L. H. V. Wang, Nat. Biotechnol. 2006, 24, 848 .

[65] W. S. Guo, X. L. Sun, O. Jacobson, X. F. Yan, K. Min, A. Srivatsan, G. Niu, D. O. Kiesewetter, J. Chang, X. Y. Chen, ACS Nano 2015, 9, 488.

[66] O. Frank, L. Kavan, M. Kalbac, Nanoscale 2014, 6, 6363.

[67] M. Yang, K. Cheng, S. B. Qi, H. G. Liu, Y. X. Jiang, H. Jiang, J. B. Li, K. Chen, H. M. Zhang, Z. Cheng, Biomaterials 2013, 34, 2796.

[68] a) K. Yang, L. Z. Feng, X. Z. Shi, Z. Liu, Chem. Soc. Rev. 2013, 42, 530; b) L. Y. Feng, L. Wu, X. G. Qu, Adv. Mater. 2013, 25, 168.

[69] V. K. Rana, M. C. Choi, J. Y. Kong, G. Y. Kim, M. J. Kim, S. H. Kim, S. Mishra, R. P. Singh, C. S. Ha, Macromol. Mater. Eng. 2011, 296, 131.

[70] G. Chauhan, V. Chopra, A. Tyagi, G. Rath, R. K. Sharma, A. K. Goyal, Eur. J. Pharm. Sci. 2017, 96, 351.

[71] a) X. M. Sun, Z. Liu, K. Welsher, J. T. Robinson, A. Goodwin, S. Zaric, H. J. Dai, Nano Res. 2008, 1, 203; b) L. M. Zhang, J. G. Xia, Q. H. Zhao, L. W. Liu, Z. J. Zhang, Small 2010, 6, 537.

[72] Z. Liu, J. T. Robinson, X. M. Sun, H. J. Dai, J. Am. Chem. Soc. 2008, 130, 10876.

[73] X. Y. Yang, X. Y. Zhang, Z. F. Liu, Y. F. Ma, Y. Huang, Y. Chen, J. Phys. Chem. C 2008, 112, 17554.

[74] X. C. Wang, K. Maeda, A. Thomas, K. Takanabe, G. Xin, J. M. Carlsson, K. Domen, M. Antonietti, Nat. Mater. 2009, 8, 76.

[75] L. S. Lin, Z. X. Cong, J. Li, K. M. Ke, S. S. Guo, H. H. Yang, G. N. Chen, J. Phys. Chem. B 2014, 2, 1031.

[76] Y. Chen, D. L. Ye, M. Y. Wu, H. R. Chen, L. L. Zhang, J. L. Shi, L. Z. Wang, Adv. Mater. 2014, 26, 7019.

[77] Y. Wang, K. Y. Wang, J. F. Zhao, X. G. Liu, J. Bu, X. Y. Yan, R. Q. Huang, J. Am. Chem. Soc. 2013, 135, 4799.

[78] T. Ren, L. Li, X. Cai, H. Dong, S. Liu, Y. Li, Polym. Chem-UK. 2012, 3, 2561.

[79] K. Ladewig, Z. P. Xu, G. Q. Lu, Exp. Opin. Drug. Deliv. 2009, 6, 907.

[80] a) S. Y. Kwak, Y. J. Jeong, J. S. Park, J. H. Choy, Solid State Ionics 2002, 151, 229; b) Z. Y. Kou, X. Wang, R. S. Yuan, H. B. Chen, Q. M. Zhi, L. Gao, B. Wang, Z. J. Guo, X. F. Xue, W. Cao, L. Guo, Nanoscale Res. Lett. 2014, 9, 587.

[81] a) B. Chen, M. Liu, L. Zhang, J. Huang, J. Yao, Z. Zhang, J. Mater. Chem. 2011, 21, 7736; b) X. Liu, D. Ma, H. Tang, L. Tan, Q. Xie, Y. Zhang, M. Ma, S. Yao, ACS Appl. Mater. Inter. 2014, 6, 8173.

[82] a) H. Bao, Y. Pan, Y. Ping, N. G. Sahoo, T. Wu, L. Li, J. Li, L. H. Gan, Small 2011, 7, 1569; b) J. Kim, H. Kim, W. J. Kim, Small 2016, 12, 1184.

[83] C. Zhang, Y. Yong, L. Song, X. Dong, X. Zhang, X. Liu, Z. Gu, Y. Zhao, Z. Hu, Adv. Healthc. Mater. 2016, 5, 2776.

[84] H. Kim, R. Namgung, K. Singha, I.-K. Oh, W. J. Kim, Bioconjugate Chem. 2011, 22, 2558.

[85] E. S. Shibu, M. Hamada, N. Murase, V. Biju, J. Photoch. Photobio. C 2013, 15, 53.

[86] X. H. Huang, P. K. Jain, I. H. El-Sayed, M. A. El-Sayed, Laser. Med. Sci. 2008, 23, 217.

[87] J. T. Robinson, S. M. Tabakman, Y. Y. Liang, H. L. Wang, H. S. Casalongue, D. Vinh, H. J. Dai, J. Am. Chem. Soc. 2011, 133,6825 .

[88] O. Akhavan, E. Ghaderi, Small 2013, 9, 3593.

[89] O. Akhavan, E. Ghaderi, S. Aghayee, Y. Fereydooni, A. Talebi, J. Mater. Chem. 2012, 22, 13773.
[90] J. W. Bai, X. Zhong, S. Jiang, Y. Huang, X. F. Duan, Nat. Nanotechnol. 2010, 5, 190.

[91] a) Z. M. Markovic, L. M. Harhaji-Trajkovic, B. M. Todorovic-Markovic, D. P. Kepic, K. M. Arsikin, S. P. Jovanovic, A. C. Pantovic, M. D. Dramicanin, V. S. Trajkovic, Biomaterials 2011, 32, 1121; b) K. Yang, L. L. Hu, X. X. Ma, S. Q. Ye, L. Cheng, X. Z. Shi, C. H. Li, Y. G. Li, Z. Liu, Adv. Mater. 2012, 24, 1868.

[92] T. P. Shao, J. Wen, Q. Zhang, Y. W. Zhou, L. Liu, L. H. Yu Wen, Y. Tian, Y. L. Zhang, W. Tian, Y. Y. Su, Z. G. Teng, G. M. Lu, J. Xu, J. Mater. Chem. B 2016, 4, 7708.

[93] T. Liu, C. Wang, W. Cui, H. Gong, C. Liang, X. Z. Shi, Z. W. Li, B. Q. Sun, Z. Liu, Nanoscale 2014, 6, 11219.

[94] S. Wang, K. Li, Y. Chen, H. Chen, M. Ma, J. Feng, Q. Zhao, J. Shi, Biomaterials 2015, 39, 206.

[95] Q. Liu, C. Y. Sun, Q. He, A. Khalil, T. Xiang, , D. B. Liu, Y. Zhou, J. Wang, L. Song, Nano Res. 2015, 8, 3982.

[96] Y. Yong, L. J. Zhou, Z. J. Gu, L. Yan, G. Tian, X. P. Zheng, X. D. Liu, X. Zhang, J. X. Shi, W. S. Cong, W. Y. Yin, Y. L. Zhao, Nanoscale 2014, 6, 10394.

[97] a) Z. L. Li, Y. Hu, K. A. Howard, T. T. Jiang, X. L. Fan, Z. H. Miao, Y. Sun, F. Besenbacher, M. Yu, ACS Nano 2016, 10, 984; b) Y. Min, G. Park, B. Kim, A. Giri, J. Zeng, J. W. Roh, S. I. Kim, K. H. Lee, U. Jeong, ACS Nano 2015, 9, 6843.

[98] W. L. Song, P. Wang, L. Cao, A. Anderson, M. J. Meziani, A. J. Farr, Y. P. Sun, Angew. Chem. Int. Ed. 2012, 51, 6498.

[99] a) P. Huang, C. Xu, J. Lin, C. Wang, X. S. Wang, C. L. Zhang, X. J. Zhou, S. W. Guo, D. X. Cui, Theranostics 2011, 1, 240; b) Y. C. Wei, F. F. Zhou, D. Zhang, Q. Chen, D. Xing, Nanoscale 2016, 8, 3530 .

[100] I. Diamond, R. Jaenicke, C. B. Wilson, A. F. Mcdonagh, S. Nielsen, S. G. Granelli, Lancet 1972, 2, 1175.

[101] a) P. R. Wei, Y. Kuthati, R. K. Kankala, C. H. Lee, Int. J. Mol. Sci. 2015. 16, 20943; b) L. Jia, L. Ding, J. W. Tian, L. Bao, Y. P. Hu, H. X. Ju, J. S. Yu, Nanoscale 2015. 7, 15953.

[102] K. Khorsandi, R. Hosseinzadeh, M. Fateh, RSC Adv. 2015, 5, 93987.

[103] Z. Hu, J. Li, C. Y. Li, S. J. Zhao, N. Li, Y. F. Wang, F. Wei, L. Chen, Y. D. Huang, J. Mater. Chem. B 2013, 1, 5003.

[104] Z. G. Wang, R. Ma, L. Yan, X. F. Chen, G. Y. Zhu, Chem. Commun. 2015, 51, 11587.

[105] J. B. Cao, H. Q. An, X. L. Huang, G. F. Fu, R. Q. Zhuang, L. Zhu, J. Xie, F. Zhang, Nanoscale 2016, 8, 10152.

[106] a) R. R. Xing, T. F. Jiao, Y. M. Liu, K. Ma, Q. L. Zou, G. H. Ma, X. H. Yan, Polymers 2016, 8; b) P. R. Wei, Y. Kuthati, R. K. Kankala, C. H. Lee, Int. J. Mol. Sci. 2015, 16, 20943.

[107] a) X. T. Liu, X. Y. Mu, X. L. Wu, L. X. Meng, W. B. Guan, Y. Q. Ma, H. Sun, C. J. Wang, X. F. Li, Biomed. Environ. Sci. 2014, 27, 676; b) J. Jeong, H. J. Cho, M. Choi, W. S. Lee, B. H. Chung, J. S. Lee, Carbon 2015, 93, 431.

[108] A. Sasidharan, S. Swaroop, C. K. Koduri, C. M. Girish, P. Chandran, L. S. Panchakarla, V. H. Somasundaram, G. S. Gowd, S. Nair, M. Koyakutty, Carbon 2015, 95, 511.

[109] L. Mao, M. J. Hu, B. C. Pan, Y. C. Xie, E. J. Petersen, Part. Fiber. Toxicol. 2016, 13, 1.

[110] K. Wang, J. Ruan, H. Song, J. L. Zhang, Y. Wo, S. W. Guo, D. X. Cui, Nanoscale Res. Lett. 2011, 6, 8.

[111] a) S. J. Choi, J. H. Choy, Nanomedicine 2011, 6, 803; b) J. Ma, R. Liu, X. Wang, Q. Liu, Y. N. Chen, R. P. Valle, Y. Y. Zuo, T. Xia, S. J. Liu, ACS Nano 2015, 9, 10498.

[112] P. R. Wei, S. H. Cheng, W. N. Liao, K. C. Kao, C. F. Weng, C. H. Lee, J. Mater. Chem. 2012, 22, 5503.

[113] M. Xu, J. Q. Zhu, F. F. Wang, Y. J. Xiong, Y. K. Wu, Q. Q. Wang, J. Weng, Z. H. Zhang, W. Chen, S. J. Liu, ACS Nano 2016, 10, 3267.

[114] S. Kanakia, J. D. Toussaint, S. M. Chowdhury, T. Tembulkar, S. Lee, Y. P. Jiang, R. Z. Lin, K. R. Shroyer, W. Moore, B. Sitharaman, Biomaterials 2014, 35, 7022. 
[115] a) J. K. Kim, J. H. Shin, J. S. Lee, J. H. Hwang, J. H. Lee, J. E. Baek, T. G. Kim, B. W. Kim, J. S. Kim, G. H. Lee, K. Ahn, S. G. Han, D. Bello, I. J. Yu, Nanotoxicology 2016, 10, 891; b) S. Xu, Z. Y. Zhang, M. Q. Chu, Biomaterials 2015, 54, 188.

[116] a) B. Li, J. Z. Yang, Q. Huang, Y. Zhang, C. Peng, Y. J. Zhang, Y. He, J. Y. Shi, W. X. Li, J. Hu, C. H. Fan, NPG Asia Mater. 2013, 5, e44; b) Z. Li, Y. X. Geng, X. Y. Zhang, W. Qi, Q. H. Fan, Y. Li, Z. X. Jiao, J. J. Wang, Y. Q. Tang, X. J. Duan, W. S. Wu, J. Nanopart. Res. 2011, 13, 2939.

[117] O. Akhavan, E. Ghaderi, A. Akhavan, Biomaterials 2012, 33 , 8017.

[118] G. Prencipe, S. M. Tabakman, K. Welsher, Z. Liu, A. P. Goodwin, L. Zhang, J. Henry, H. J. Dai, J. Am. Chem. Soc. 2009, 131, 4783.

[119] K. Yang, J. M. Wan, S. Zhang, Y. J. Zhang, S.-T. Lee, Z. Liu, ACS Nano 2011, 5, 516.

[120] A. H. Hung, R. J. Holbrook, M. W. Rotz, C. J. Glasscock, N. D. Mansukhani, K. W. MacRenaris, L. M. Manus, M. C. Duch, K. T. Dam, M. C. Hersam, T. J. Meade, ACS nano 2014, 8, 10168.

[121] J. Nunnari, A. Suomalainen, Cell 2012, 148, 1145.

[122] A. Mallick, P. More, S. Ghosh, R. Chippalkatti, B. A. Chopade, M. Lahiri, S. Basu, Acs. Appl. Mater. Inter. 2015, 7, 7584.

[123] A. Chakraborty, N. R. Jana, J. Phys. Chem. C 2015, 119, 2888.

[124] K. Yang, H. Gong, X. Z. Shi, J. M. Wan, Y. J. Zhang, Z. Liu, Biomaterials 2013, 34, 2787.
[125] N. Kurantowicz, B. Strojny, E. Sawosz, S. Jaworski, M. Kutwin, M. Grodzik, M. Wierzbicki, L. Lipinska, K. Mitura, A. Chwalibog, Nanoscale Res. Lett. 2015, 10, 1.

[126] D. A. Jasim, C. Menard-Moyon, D. Begin, A. Bianco, K. Kostarelos, Chem. Sci. 2015, 6, 3952.

[127] J. H. Liu, S. T. Yang, H. F. Wang, Y. L. Chang, A. N. Cao, Y. F. Liu, Nanomedicine 2012, 7, 1801.

[128] H. Pieper, S. Chercheja, S. Eigler, C. E. Halbig, M. R. Filipovic, A. Mokhir, Angew. Chem. Int. Ed. 2016, 55, 405.

[129] M. H. Yang, S. Q. Gong, Chem. Commun. 2010, 46, 5796.

[130] S. Y. Lee, S. H. Kim, H. Kawai, H. Lee, G. Lee, Y. Park, New J. Chem. 2014, 38, 2225.

[131] C. E. Ashley, E. C. Carnes, G. K. Phillips, D. Padilla, P. N. Durfee, P. A. Brown, T. N. Hanna, J. W. Liu, B. Phillips, M. B. Carter, N. J. Carroll, X. M. Jiang, D. R. Dunphy, C. L. Willman, D. N. Petsev, D. G. Evans, A. N. Parikh, B. Chackerian, W. Wharton, D. S. Peabody, C. J. Brinker, Nat. Mater. 2011, 10, 476.

[132] G. P. Kotchey, B. L. Allen, H. Vedala, N. Yanamala, A. A. Kapralov, Y. Y. Tyurina, J. Klein-Seetharaman, V. E. Kagan, A. Star, ACS Nano 2011, 5, 2098.

[133] G. S. Song, J. L. Hao, C. Liang, T. Liu, M. Gao, L. Cheng, J. Q. Hu, Z. Liu, Angew. Chem. Int. Ed. 2016, 55, 2122.

Received: October 14, 2016 Revised: November 27, 2016 Published online: January 11, 2017 